\title{
Pneumomediastinum and subcutaneous emphysema after noninvasive ventilation in a COVID-19 patient
}

\author{
Natale Vazzana, Silvia Ognibene, Francesco Dipaola \\ Department of Internal Medicine, C. Magati Hospital, Scandiano, AUSL Reggio Emilia, Italy
}

An 80-year-old man was admitted because of persistent fever and dyspnea. Two days before he presented at the emergency department with a 5-day history of non-productive cough and fever. Chest X-ray revealed bilateral alveolar infiltrates. Blood tests showed mild leukocytosis, increased C-reactive protein and normal procalcitonin. Oxygen saturation was $96 \%$ at ambient air. High-resolution chest tomography (HRCT) showed bilateral ground-glass opacities. SARS-CoV-2 infection was diagnosed via nucleic acid test on nasopharyngeal swab. Hydroxychloroquine was started and he was discharged to home isolation. In the following 48 hours his symptoms did not improve. At second presentation, oxygen saturation was decreased to 93\%, body temperature was $39.9^{\circ} \mathrm{C}$, and respiratory rate was 30 breaths/min. Physical examination was notable for crackles in both lung bases. He was admitted to our COVID-19 ward and supplemented with oxygen through a non-rebreather face mask. Antiviral therapy (lopinavir/ritonavir) was added to ongoing hydroxychloroquine for 10 days. Intravenous (IV) antibiotics (ceftriaxone plus azithromycin) were also administered from day (D)2 to D7, and ceftriaxone alone was extended until D12. He was also receiving thromboprophylaxis (fondaparinux $2.5 \mathrm{mg} / \mathrm{day}$ ) since admission. Although fever decreased within the first 5 days, his respiratory status progressively worsened, with a decrease in PaO2/FiO2 to less than 150. Steroid was started at D6 (IV methylpredinisolone $60 \mathrm{mg} / \mathrm{die}$ ) with little improvement. Noninvasive ventilation was then applied (PPEP 6-8 cmH2O), but it was stopped after 48 hours because of poor compliance. Repeated HRCT scan showed increased ground-glass opacities and parenchymal consolidations (Figure 1). Pneumomediastinum was also observed, together with subcutaneous emphysema (Figure 1). Conservative treatment was applied, and antibiotic therapy was resumed because of fever relapse. In the following days, he received conventional oxygen supplementation, fever disappeared, but no further improvement of respiratory function was observed. He was ultimately transferred to a long-term care unit, where he continued receiving supportive and palliative treatment.
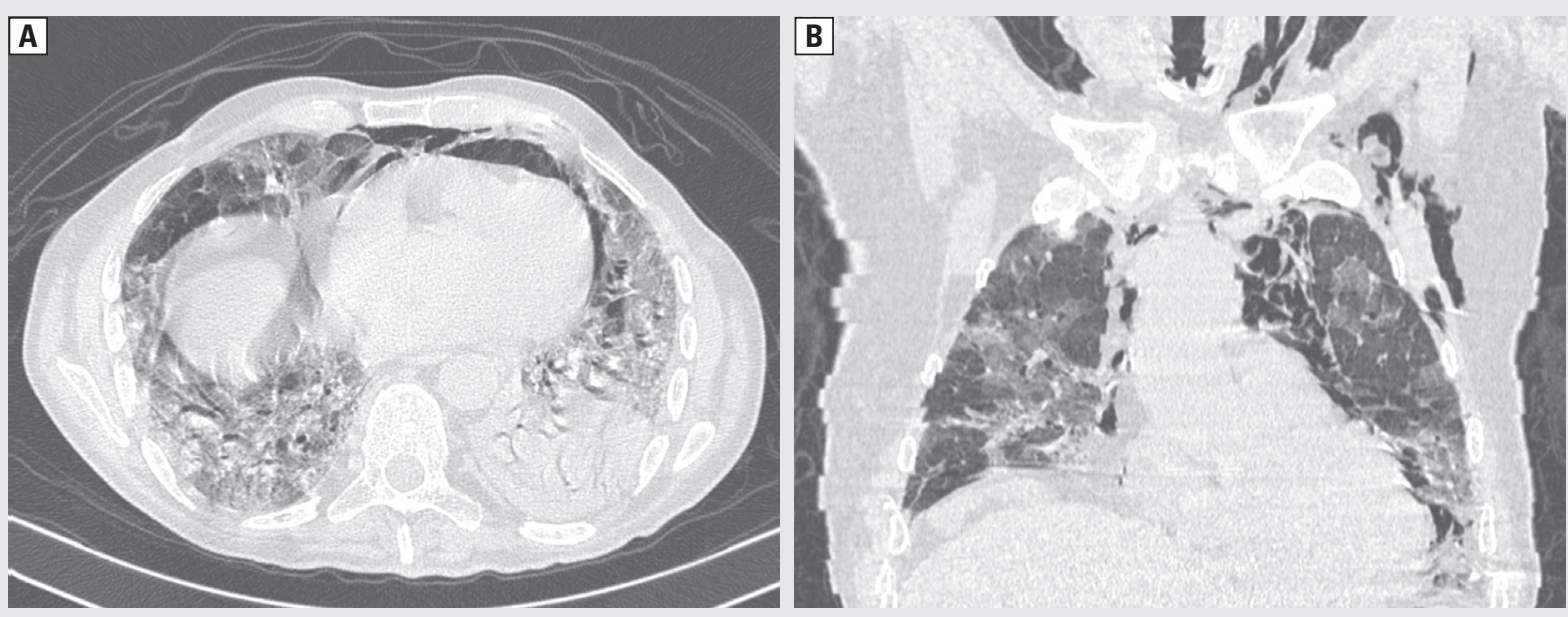

Figure 1. A. HRCT scan showing multiple ground-glass opacities with thickening of interlobular and intralobular septa (crazy-paving pattern) and a parenchymal consolidation in the left lower lobe. Pneumomediastinum was also observed around the heart, with the presence of connective tissue septae; B. Pneumomediastinum extended upward between the mediastinal pleura, the aortic arch and the superior mediastinum, together with subcutaneous emphysema extending to the soft tissue of the cervico-axillary region, and the lateral thoracic wall

Address for correspondence: Natale Vazzana, Department of Internal Medicine, C. Magati Hospital, AUSL Reggio Emilia, Italy; e-mail: natale.vazzana@ausl.re.it DOI: 10.5603/ARM.a2020.0141

Received: 18.04.2020

Copyright (C) 2020 PTChP

ISSN 2451-4934

Conflict of interest: None declared 
Pneumomediastinum is an uncommon complication of mechanical ventilation [1]. Spontaneous pneumomediastinum has also been observed in association with several structural lung diseases, including chronic obstructive lung disease, asthma, and interstitial lung diseases [1, 2]. More recently it has been reported complicating the course of COVID-19 [3]. Both spontaneous and ventilation-associated pneumomediastinum has been observed in patients with SARS, with high peak LDH level associated with its development [4]. In the presence of diffuse alveolar damage, free air could leak from ruptured alveoli, dissecting along the bronchovascular sheaths towards the mediastinum (Macklin effect) [1]. Symptoms and signs include chest pain, worsening dyspnea, subcutaneous emphysemas, mediastinal (Hamman's) crunch on heart auscultation [1]. Pneumomediastinum is generally a self-limiting condition [2]. More rarely it could be life-threatening by mimicking cardiac tamponade [1]. Here, we reported a case of pneumomediastinum in a patient with severe COVID-19-related pneumonia necessitating noninvasive ventilation. Prompt recognition is required during ventilatory support as it may promote its progression [5]. In both SARS and COVID-19, pneumomediastinum has been associated with a more severe course [3,4], possibly reflecting massive alveolar and interstitial pulmonary injury. The occurrence rate of pneumomediastinum among ventilated COVID-19 patients is still unknown. Further data are needed to identify the mechanisms, frequency, risk factors and prognostic role of this rare complication of COVID-19.

\section{References:}

1. Kouritas VK, Papagiannopoulos K, Lazaridis G, et al. Pneumomediastinum. J Thorac Dis. 2015; 7(Suppl 1): S44-S49, doi: 10.3978/i. issn.2072-1439.2015.01.11, indexed in Pubmed: 25774307.

2. Iyer VN, Joshi AY, Ryu JH. Spontaneous pneumomediastinum: analysis of 62 consecutive adult patients. Mayo Clin Proc. 2009; 84(5): 417-421, doi: 10.1016/S0025-6196(11)60560-0, indexed in Pubmed: 19411438.

3. Kong N, Gao C, Xu MS, et al. COVID-19 with spontaneous pneumomediastinum. Lancet Infect Dis. 2020; 20(4): 510-3577, doi: 10.1016/S1473-3099(20)30156-0, indexed in Pubmed: 32164830.

4. Chu CM, Leung YY, Hui JYH, et al. Spontaneous pneumomediastinum in patients with severe acute respiratory syndrome. Eur Respir J. 2004; 23(6): 802-804, doi: 10.1183/09031936.04.00096404, indexed in Pubmed: 15218989.

5. Ruggeri P, Girbino G. Fatal pneumomediastinum associated with use of noninvasive mechanical ventilation. Respirol Case Rep. 2014; 2(4): 126-128, doi: 10.1002/rcr2.73, indexed in Pubmed: $\underline{25530859}$. 